unipolar disorder developed dementia (MMSE $<24)$ compared with $3.4 \%$ of age-matched controls (Kessing, 1998; Kessing et al, 1999). Even within a younger sample of psychiatric patients (approximate mean age 50 years), Kessing et al (1999) reported that people with bipolar disorder had the highest risk of receiving a diagnosis of dementia, followed by those with unipolar affective disorder, schizophrenia and neuroses. Thus, if affective disorders do increase both the risk of dementia and the likelihood of receiving lithium treatment, then owing to the sampling method used by Dunn et al one could expect to find more lithium treatment among elderly people with dementia. Dunn et al discussed this alternative explanation of their findings as a 'reverse causation' possibility.

Dr Terao mentions the possible effects of lithium on GSK-3 beta. We recently investigated the effects of lithium on the transcriptional regulation of GSK-3 beta and found a significant reduction of its expression in primary cultures of rat hippocampal neurons as well as a reduction in regional intracerebral expression in lithium-treated adult rats and in leukocytes of elderly patients undergoing chronic lithium therapy for bipolar disorder (details available from the authors). These observations suggest a mechanism for GSK-3 beta inhibition by lithium, which may influence the formation of both amyloid plaques and neurofibrillary tangles, the two neuropathological hallmarks of Alzheimer's disease.

We think that it is important to investigate further the potential protective effect of lithium in Alzheimer's disease, as this could represent a low-cost universally available strategy to reduce the prevalence.

Dunn, N., Holmes, C. \& Mullee, M. (2005) Does lithium therapy protect against the onset of dementia? Alzheimer Disease and Associated Disorders, 19, 20-22.

Kessing, I. V. (1998) Cognitive impairment in the euthymic phase of affective disorder. Psychological Medicine, 28, 1027-1038.

Kessing, I. V., Olsen, E. W., Mortensen, P. B., et al (1999) Dementia in affective disorder: a case-register study. Acta Psychiatrica Scandinavica, 100, 176-185.

Nilsson, F. M., Kessing, I. V., Sorensen, T. M., et al (2002) Enduring increased risk of developing depression and mania in patients with dementia. Journal of Neurology, Neurosurgery and Psychiatry, 73, 40-44.

W. F. Gattaz Institute of Psychiatry, University of São Paulo, Brazil. Email: gattaz@usp.br

O.V. Forlenza, P.V. Nunes Institute of Psychiatry, University of São Paulo, Brazil doi: 10.1192/bjp.191.4.36la

\section{Mortality and electroconvulsive therapy}

Munk-Olsen et al (2007) reported that the mortality rate from natural causes was lower for patients undergoing electroconvulsive therapy (ECT) than for other psychiatric in-patients. The lower relative risk was particularly significant for mortality linked to respiratory disease $(\mathrm{RR}=0.67$ $95 \%$ CI 0.55-0.95) and a trend was founded for cardiovascular disease $(\mathrm{RR}=0.85,95 \%$ CI $0.70-1.03)$. The authors concluded that this decreased risk of mortality from natural causes is unlikely to be the result of a selection bias. They based this statement on: (a) the absence of absolute contraindications to ECT in the international guidelines; and (b) the concordant findings of previous studies.

At variance with this statement, clinical practice suggests that psychiatrists are generally reluctant to consider ECT in patients with medical illness, and are more likely to ask for the opinion of a colleague in such a case (e.g. anaesthetist, cardiologist) (Benbow \& Shah, 2002). Thus, patients with severe medical illness could be less likely to be treated by ECT. Furthermore, identification of cardiovascular diseases or pulmonary disorders, as well as physical examination and standard laboratory tests are part of a systematic screening procedure before ECT. This practice improves the diagnosis and the treatment of medical comorbidities. Indeed, the absence of such preliminary medical examination led to a high level of cardiac complications after ECT in the past (Gerring \& Shields, 1982).

Accordingly current guidelines emphasise the importance of identifying and carefully managing patients with risk factors before, during and after ECT, as well as assessing the risks associated with anaesthesia (National Institute for Clinical Excellence, 2003). Patients receiving ECT are therefore not representative of all psychiatric in-patients. The careful assessment and treatment of their physical comorbidities contrasts with the increased rate of untreated physical illness in psychiatric patients, mostly because of inadequate somatic care in psychiatric units (Rasanen et al, 2006). Therefore, the observed diminution of mortality from natural causes in patients with ECT is more likely to be related to appropriate medical assessment and treatment than to a direct effect of ECT on physical health.
Benbow, S. M. \& Shah, A. (2002) A survey of the views of geriatric psychiatrists in the United Kingdom on the use of electroconvulsive therapy to treat physically ill people. International Journal of Geriatric Psychiatry, I7, 956-961.

Gerring, J. P. \& Shields, H. M. (1982) The identification and management of patients with a high risk for cardiac arrhythmias during modified ECT. Journal of Clinical Psychiatry, 43, 140-143.

Munk-OIsen, T., Laursen, T. M., Videbech, P., et of (2007) All-cause mortality among recipients of electroconvulsive therapy. Register-based cohort study. British Journal of Psychiatry, 190, 435-439.

National Institute for Clinical Excellence (2003) The Clinical Effectiveness and Cost Effectiveness of Electroconvulsive Therapy (ECT) for Depressive Illness, Schizophrenia, Catatonia and Mania. NICE.

Rasanen, S., Meyer-Rochow, V. B., Moring, J., et al (2006) Hospital-treated physical illnesses and mortality: an II-year follow-up study of long-stay psychiatric patients. European Psychiatry, 22, 21I-218.

Y. Le Strat, P. Gorwood Assistance

Publique Hôpitaux de Paris, Paris, France. Email: philip.gorwood@Imr.aphp.fr

doi: 10.I192/bjp.191.4.362

In an era that has seen ECT being opposed for political not clinical reasons, it was heartening to see an article on ECT addressing the very important issue of mortality. The study of Munk-Olsen et al (2007) is based on the Danish registry system which is acclaimed for its reliability, but certain issues need further clarification. It would have been relevant to know the total number of patients who received ECT and the total number of ECT treatments received by patients over the study period. Furthermore, the results could be better understood if information regarding physical comorbidity and the age of patients at the time of ECT had been provided. These variables can have a strong influence on mortality rates. In addition, as the study included only in-patients it is likely that the sample included patients who were severely ill. Also, the results show that inclusion of 'days since last ECT treatment' in the analysis causes the relative risk of mortality from natural causes of patients 'discharged within the past $8-30$ days' to rise.

The relative risk of mortality from natural causes is also highest within 7 days of last ECT ( $R R=2.11)$, which is similar to the trend seen in deaths due to unnatural causes, especially suicide. Both these figures go against the conclusion of the authors that the mortality from natural causes is lower with ECT. It must also be noted that the relative risk of dying by suicide after 